\title{
REVISIÓN DE LA LITERATURA EN EL DIAGNÓSTICO IMAGENOLÓGICO DEL QUISTE DENTÍGERO
}

\author{
REVIEW OF THE LITERATURE IN THE IMAGENOLOGICAL DIAGNOSIS OF THE \\ DENTIGEROUS CYST \\ Rubén Alfredo Castro Pesce \\ castropesce@hotmail.com \\ ORCID: 0000-0002-3073-7498
}

\section{RESUMEN}

El quiste dentígero, incluido dentro de la clasificación de los quistes odontogénicos por la OMS en el 2017, constituye una patología relevante en el área buco maxilofacial. Si bien su crecimiento es lento y asintomático, cuando alcanza grandes dimensiones, tiene capacidad para provocar destrucción ósea en los maxilares, lo que ocasiona alteraciones estéticas y funcionales. Además, presenta una alta prevalencia, con el quiste odontogénico como el más común, después del quiste radicular inflamatorio. La radiología representa una herramienta fundamental en su diagnóstico y muchas veces su detección constituye un hallazgo radiográfico. Normalmente, se emplean técnicas en 2D como la radiografía periapical, panorámica y oclusal, las cuales cumplen un rol muy importante en su detección y diagnóstico. No obstante, debe considerarse que la similitud del quiste dentígero con otros procesos patológicos, tanto a nivel clínico como radiográfico, muchas veces lleva a un diagnóstico y tratamiento inadecuado.
Con este trabajo se pretende evidenciar si el advenimiento de nuevas tecnologías como la resonancia magnética y la tomografía computarizada de haz cónico permiten mejorar la posibilidad del diagnóstico del quiste dentígero y distinguirlo de otras lesiones o patologías con las cuales se realiza el diagnóstico diferencial.

Palabras clave: quiste dentígero, radiografía convencional, tomografía computarizada, diagnóstico diferencial, resonancia magnética, quistes odontogénicos

\section{ABSTRACT}

The dentigerous cyst, included in the classification of odontogenic cysts according to the WHO year 2017, constitutes a relevant pathology in the maxillofacial buccal area. Although its growth is slow and asymptomatic, when it reaches large dimensions it has the capacity to cause bone destruction in the jaws, causing aesthetic and functional alterations. In

Citar como: Castro Pesce RA. Revisión de la literatura en el diagnóstico imagenológico del quiste dentígero. Rev Cient Odontol (Lima). 2019; 7 (2): 108-118.

${ }^{1}$ Cirujano Dentista Universidad de Talca, Chile. 
addition, it has a high prevalence, being the most common odontogenic cyst, after the inflammatory root cyst. Radiology represents a fundamental tool in its diagnosis and often its detection constitutes a radiographic finding. Normally 2D techniques such as periapical, panoramic and occlusal radiography are used, which play a very important role in their detection and diagnosis. However, it should be considered that the similarity of the dentigerous cyst with other pathological processes, both clinically and radiographically, often leads to inadequate diagnosis and treatment. This work is intended to show whether the advent of new technologies such as magnetic resonance imaging and conical beam computed tomography, allow to improve the possibility of diagnosis of the dentigerous cyst and distinguish it from other lesions or pathologies with which a differential diagnosis is made.

Keywords: dentigerous cyst, conventional radiography, computed tomography, differential diagnosis, magnetic resonance, odontogenic cysts

\section{INTRODUCCIÓN}

Entre las patologías inherentes al territorio bucomaxilofacial, los quistes odontogénicos constituyen un grupo de lesiones que afecta a los maxilares y puede ocasionar alteraciones estéticas y funcionales con diferentes grados de severidad. Además, las similitudes desde el punto de vista clínico y radiográfico que presentan las distintas formaciones quísticas pueden llevar a un diagnóstico y tratamiento inadecuado y dar como resultado recidiva de estas. Por lo tanto, es muy importante realizar un diagnóstico correcto y oportuno que permita lograr un tratamiento efectivo $\left(^{1-6}\right)$.

El quiste es una cavidad anormal, de contenido líquido o semilíquido, formado por una pared de tejido conectivo y revestido internamente por epitelio. Se caracteriza por ser asintomático $\mathrm{y}$, muchas veces, se detecta a través de un examen radiográfico de rutina. Su denominación como quiste odontogénico responde a la persistencia de restos epiteliales provenientes del proceso de formación de la pieza dentaria. Dicho epitelio representa el punto de partida en su desarrollo $\left(^{(-10}\right)$.

Con relación a la manera de clasificarlos, esta ha presentado distintas modificaciones con los años. Estos cambios obedecen al comportamiento clínico y la reactividad ante ciertos marcadores que permiten determinar la capacidad proliferativa del epitelio. Además, existen marcadores de transformación tumoral, en los cuales también se han basado para realizar modificaciones en su clasificación $\left({ }^{11-13}\right)$.

Los quistes odontogénicos se caracterizan por presentar un crecimiento expansivo, lento y con destrucción del hueso de los maxilares, y pueden alcanzar grandes dimensiones si no se diagnostican precozmente. Entre ellos, el quiste dentígero es uno de los más comunes, con una prevalencia del $26,6 \%$. Es más frecuente encontrarlos en el maxilar inferior, asociados principalmente con el tercer molar incluido, pero pueden asociarse también con otras piezas dentarias. Se ubican a nivel cervical de la pieza incluida y presentan, además, tres variantes radiográficas importantes de reconocer para determinar si en realidad la imagen corresponde a un quiste dentígero $\left({ }^{13-15}\right)$.

La radiología juega un rol fundamental en su detección oportuna, ya que constituye el primer paso para el diagnóstico de los quistes. Existen diferentes técnicas radiográficas, entre ellas las radiografías convencionales 2D, como la periapical, la oclusal y la panorámica, siendo esta última muchas veces el estudio de 
elección, ya que nos permite observar ambos maxilares y, por lo tanto, determinar su ubicación y extensión $\left.{ }^{15-16}\right)$. También se utilizan técnicas complementarias como la proyección de Waters o la técnica oblicua lateral.

Actualmente, hay un desarrollo constante de técnicas más avanzadas, entre ellas destaca la tomografía computarizada de haz cónico, la cual representa un gran avance en el campo de la imagenología, ya que brinda imágenes en 3D de gran calidad y precisión diagnóstica.

Permite, además, realizar múltiples cortes en sentido axial, coronal y sagital, lo cual entrega más información y representa un real aporte diagnóstico. Se emplea, además, una baja dosis de radiación y con un corto tiempo de exposición $\left({ }^{15-16}\right)$. Existe también la resonancia magnética, la cual, si bien se emplea poco, ha logrado resultados promisorios.

Por lo tanto, considerando los adelantos en el campo de la radiología bucal y maxilofacial, y teniendo en cuenta la importancia que los quistes odontogénicos y, en especial, el quiste dentígero representa en el sistema estomatognático, es que consideramos relevante conocer e investigar si dichos avances en el área de la imagenología, que nos permiten determinar la ubicación exacta de la lesión y el contenido de la misma, han facilitado su detección y precisado su diagnóstico.

\section{ORIGEN E HISTOPATOLOGÍA DEL QUISTE DENTÍGERO}

Hay acuerdo en distintos autores con relación al origen de los quistes odontogénicos, el cual obedece a la persistencia o permanencia de epitelio luego del proceso de desarrollo de los tejidos dentarios. Existen diferentes restos epiteliales que dan origen a los quistes odontogénicos $\left({ }^{17,18}\right)$.

Dentro de la clasificación de la OMS de 2017, el quiste dentígero se encuentra entre los quistes odontogénicos del desarrollo, y su origen es relacionado con el epitelio reducido del órgano del esmalte posterior al proceso de amelogénesis. No obstante, la literatura postula distintas teorías para explicar su desarrollo. Una de ellas hace referencia al acúmulo de contenido líquido entre el tejido adamantino y el epitelio reducido del esmalte, o entre las capas del mismo epitelio, debido a cambios de la presión hidrostática ejercida por una pieza en proceso eruptivo. También se atribuye a su origen una alteración o degeneración del retículo estrellado durante el proceso de formación del tejido adamantino.

Otra teoría menciona un crecimiento de la pared de tejido conectivo a nivel del folículo y también se ha relacionado su origen a la presencia de piezas deciduas afectadas por un proceso inflamatorio, el cual induce la formación de la cavidad quística en la pieza definitiva. Respecto de este último caso, hay autores que sugieren la presencia del quiste dentígero del desarrollo y el quiste dentígero inflamatorio $\left({ }^{17-20}\right)$.

El quiste dentígero es una cavidad patológica revestida por epitelio escamoso estratificado no queratinizado, con dos o tres láminas de células planas y rodeada de tejido conectivo $\left({ }^{21,22}\right)$, la cual se desarrolla alrededor de la corona de un diente incluido a nivel de su límite cervical. Su crecimiento es lento y asintomático, por lo tanto, la mayoría de las veces su detección corresponde a un hallazgo radiográfico. En su interior se observa un contenido líquido o semilíquido, el cual, se cree, provoca un aumento paulatino de la presión interna que favorece su crecimiento y desarrollo $\left({ }^{20-23}\right)$. 
En una primera etapa de su crecimiento, la cual puede mantenerse varios años, no se observa ningún cambio clínico evidente. Posteriormente, a medida que avanza su desarrollo, se percibe una alteración del contorno óseo maxilar y cambios a nivel de la simetría facial. Se presenta de consistencia dura e indolora, puede desplazar estructuras vecinas y provocar rizólisis de las piezas adyacentes a la lesión. El tejido óseo circundante, incluso, se puede adelgazar hasta desaparecer y presentarse entonces fluctuante a la palpación. Finalmente, y ya en una etapa muy avanzada, se puede vaciar su contenido a través de una fístula traumática o por un proceso infeccioso. Se describen, entonces, distintas complicaciones a las cuales puede estar asociado el quiste dentígero: fractura ósea patológica, deformación del tejido óseo, pérdida de la pieza comprometida y transformación en distintas patologías como ameloblastoma, carcinoma mucoepidermoide y carcinoma de células escamosas $\left({ }^{20-23}\right)$.

\section{EPIDEMIOLOGÍA DEL QUISTE DENTÍGERO}

El quiste dentígero es uno de los más frecuentes, superado solamente por el quiste radicular. $\mathrm{Su}$ incidencia ha sido reportada en un $39,9 \%$ para el quiste radicular y un $33 \%$ para el quiste folicular. En tanto, la prevalencia ha mostrado mayor discrepancia entre los distintos autores, desde un $13,9 \%$ hasta un $26,6 \%$.

Con relación a su ubicación, es más habitual encontrarlo en el maxilar inferior y asociado al tercer molar, y en el maxilar superior es más frecuente en relación con el canino. También se ha relacionado con el tercer molar superior, el premolar inferior e, incluso, en piezas dentarias supernumerarias $\left({ }^{23,24}\right)$.
Es más frecuente en hombres que en mujeres, no presenta predilección por raza y, en cuanto a la edad, se presenta con mayor frecuencia entre los 20 y 30 años, pero también se han reportado algunos casos en niños. En general, su presentación es unilateral y los casos bilaterales se encontraron asociados con síndromes como la mucopolisacaridosis y la displasia cleidocreaneal $\left.{ }^{24-26}\right)$.

\section{VARIANTES \\ RADIOGRÁFICAS}

El estudio por imágenes nos muestra un área radiolúcida de forma redondeada, unilocular y con límites bien definidos a través de una cortical ósea y en relación con la corona de una pieza no erupcionada y a nivel cervical de la misma (figura 1).

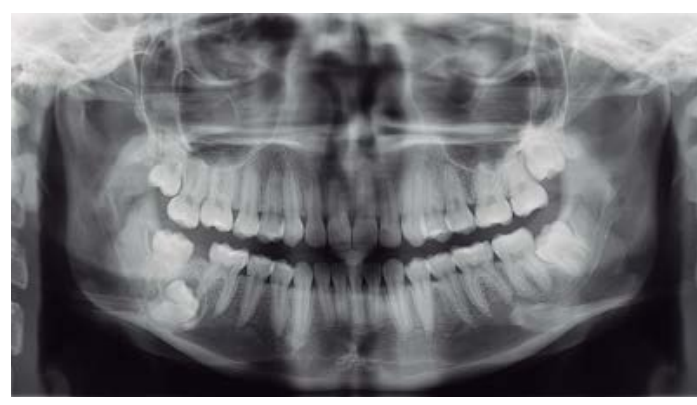

FIGURA 1. RADIOGRAFÍA PANORÁMICA QUE MUESTRA LAS CARACTERÍSTICAS IMAGENOLÓGICAS DEL QUISTE DENTÍGERO EN SU VARIEDAD CENTRAL.

Gentileza de Centro de Diagnóstico por IMÁGENES CDI-LIMA.

Se pueden identificar tres variantes radiográficas:

- Central, en la cual el quiste envuelve de forma simétrica la corona de la pieza incluida.

- Lateral, en la que el quiste se encuentra en posición lateral con relación a la corona. 
- Circunferencial, en la que se aprecia cómo el quiste envuelve completamente la pieza dentaria involucrada $\left({ }^{24-26}\right)$ (figura 2).

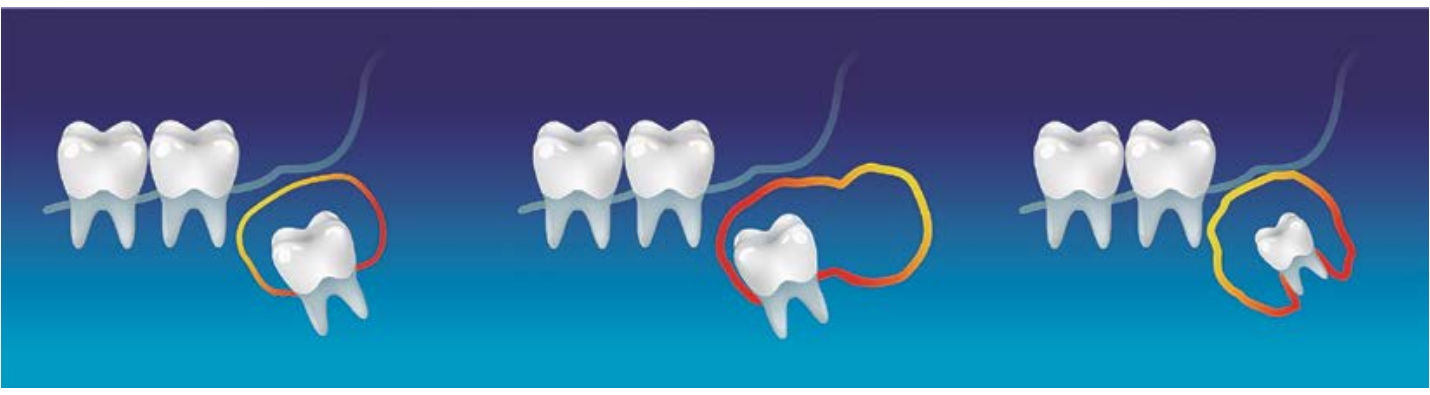

FIGURA 2. ESQUEMA CON LAS VARIANTES RADIOGRÁFICAS QUE PUEDE PRESENTAR UN QUISTE DENTÍGERO (CENTRAL, LATERAL Y CIRCUNFERENCIAL). De autoría propia.

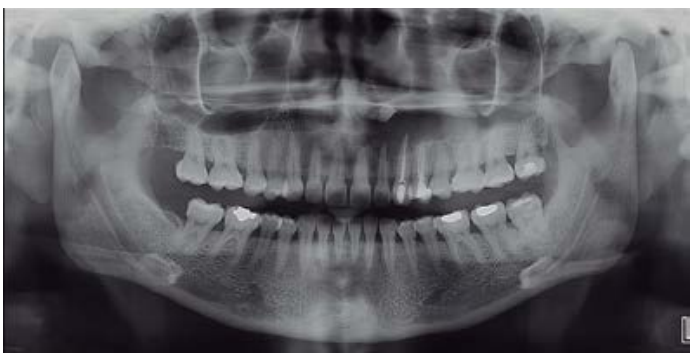

FIGURA 3. IMAGEN DE QUISTE DENTÍGERO EN RADIOGRAFÍA PANORÁMICA. Gentileza de Centro de Diagnóstico por Imágenes CDI Lima.

Consideramos importante reconocerlas y establecer la relación real con la pieza dentaria comprometida. Las imágenes en $2 \mathrm{D}$ pueden, en algunos casos, inducirnos a un error diagnóstico por proyección de la imagen y la tomografía computarizada de haz cónico puede determinar su ubicación real en relación con la pieza incluida y, de esa manera, confirmar o refutar el diagnóstico inicial.

\section{TÉCNICAS RADIOGRÁFICAS CONVENCIONALES}

Las técnicas radiográficas en 2D para el diagnóstico del quiste dentígero incluyen a la ortopantomografía, la radiografía oclusal y la periapical. La ortopantomografía constituye una muy buena técnica para el diagnóstico inicial, dado que nos brinda una visión panorámica de los

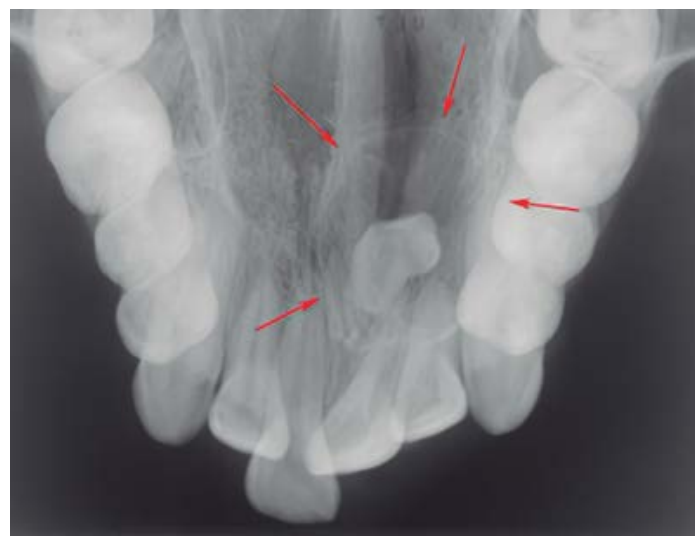

FIGURA 4. IMAGEN DE QUISTE DENTÍGERO EN RADIOGRAFÍA OCLUSAL Y PERIAPICAL. Gentileza de Centro de Diagnóstico por imágenes CDI Lima.

maxilares, lo que permite determinar la extensión y los límites del proceso quístico. La radiografía oclusal, por su parte, nos permite evaluar el compromiso del proceso palatino, como así el de las tablas óseas vestibular y lingual. La radiografía periapical, si bien nos entrega una imagen precisa y nítida del quiste, presenta limitaciones según el tamaño de la lesión y la ubicación de la pieza dentaria afectada $\left({ }^{27,}{ }^{28}\right)$ (figuras 3 y 4).

Existen también otras proyecciones entre las técnicas imagenológicas convencionales, como la técnica de Waters, empleada para el maxilar superior cuando hay compromiso de seno 
maxilar y la técnica oblicua lateral para las formaciones quísticas presentes en el maxilar inferior, las cuales se consideran técnicas complementarias $\left({ }^{27,28}\right)$.

Las radiografías periapicales y panorámicas se emplean habitualmente en la práctica clínica diaria para el diagnóstico del quiste dentígero. La radiografía panorámica nos permite, en la mayoría de los casos, descubrir la lesión en forma incidental como parte de un estudio radiográfico de rutina $\left({ }^{28}\right)$.

La radiografía retroalveolar nos entrega una imagen con detalle de la lesión; no obstante, cuando esta es extensa y compromete varias piezas dentarias y estructuras anatómicas vecinas, y cuando además es relevante determinar la naturaleza y el contenido de la imagen observada, se hace evidente la necesidad de ampliar el estudio radiográfico con el fin de obtener toda la información necesaria. $\left({ }^{28,29}\right)$.

Además, se debe tener en cuenta que la información que entrega una imagen en 2D muchas veces es limitada porque, al ser bidimensional, existe superposición de planos y efectos de distorsión y magnificación de la imagen, lo cual compromete su calidad diagnóstica y puede llevar al clínico a una elección de tratamiento equivocada $\left({ }^{29-31}\right)$.

Por lo tanto, dependiendo del caso clínico, se debe recurrir a otras técnicas por imágenes para llegar a un mejor diagnóstico que permita, a su vez, planificar y ejecutar de mejor manera el tratamiento indicado.

\section{NUEVAS TÉCNICAS IMAGENOLÓGICAS}

El advenimiento de nuevas tecnologías, como la tomografía computarizada de haz cónico, representa un paso importante en el estudio por imágenes para distintas condiciones o patologías de los maxilares.

La tomografía computarizada de haz cónico presenta distintas ventajas: entrega una imagen tridimensional, lo que permite su evaluación en cortes axiales, coronales y sagitales del área de estudio, con lo que se obtiene muy buena información dado que se eliminan las limitaciones de las imágenes en 2D, como la superposición de distintas estructuras y la distorsión que sufre la imagen. Asimismo, nos permite ubicar, dimensionar y relacionar la lesión con la pieza dentaria incluida y con estructuras vecinas (figuras 5 y 6). Nos permite evidenciar reabsorción ósea, expansión de la cortical, esclerosis del hueso que

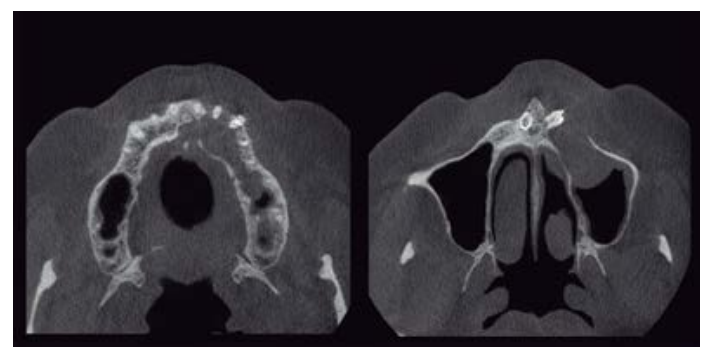

FIGURA 5. TOMOGRAFÍA COMPUTARIZADA DE HAZ CÓNICO. VISTAS AXIALES. Gentileza de Centro de Diagnóstico por imágenes CDI-Lima.

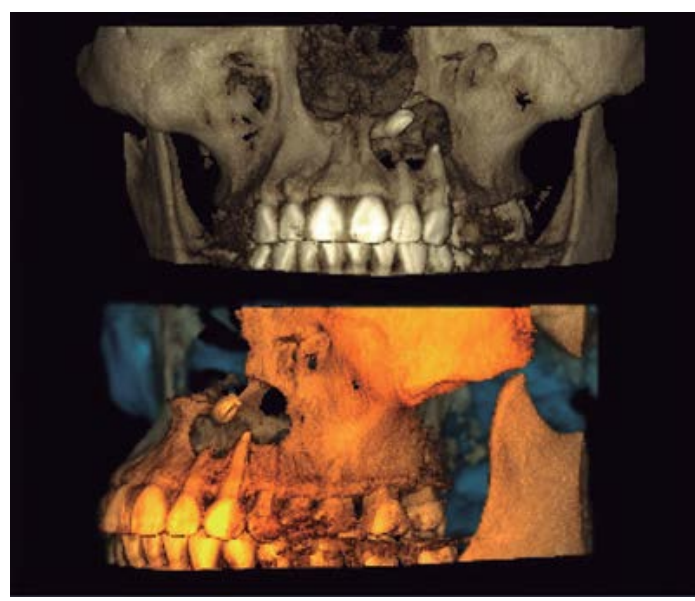

FiguRA 6. IMAGEN DE RECONSTRUCCIÓN EN 3D. Gentileza de Centro de Diagnóstico por Imágenes CDI-Lima. 
rodea la lesión y la presencia de calcificaciones internas. Brinda una gran ventaja al clínico en la planificación y el abordaje quirúrgico $\left.{ }^{32-35}\right)$.

La desventaja de este tipo de tomografía es que no puede evaluar el tejido blando y esto es una dificultad para distinguir una lesión quística de una neoplásica $\left({ }^{35}\right)$.

Existe también la resonancia magnética, la cual no se emplea habitualmente para el diagnóstico de esta patología, pero merece ser mencionada dado que no expone al paciente a la radiación ionizante y brinda información sobre los tejidos blandos que rodean la lesión, lo que permite además determinar su naturaleza y contenido, información de gran relevancia para el diagnóstico diferencial entre lesiones quísticas y neoplásicas que presentan características clínicas e imagenológicas similares, así como para planificar el tratamiento. Sus desventajas son su alto costo asociado y poca disponibilidad en la práctica clínica ${ }^{36,37}$ ).

\section{DIAGNÓSTICO IMAGENOLÓGICO DIFERENCIAL DEL QUISTE DENTIGERO}

Existen distintas entidades con las cuales el quiste dentígero guarda similitudes en su imagen radiográfica, dado que también se puede apreciar un área radiolúcida, de bordes definidos y corticalizados, y con relación a piezas dentarias incluidas. Entre ellas debemos mencionar al queratoquiste odontogénico, el quiste odontogénico ortoqueratinizado, el ameloblastoma uniquístico y el tumor odontogénico adenomatoide (TOA). Este último, especialmente cuando se presenta sin calcificaciones, es también llamado folicular, y además se pueden diferenciar porque el TOA se extiende a lo largo de la raíz ${ }^{(38,39}$ ).
Debemos recordar que, si bien el quiste dentígero presenta generalmente una imagen unilocular, cuando se desarrolla y alcanza grandes dimensiones, puede presentar un aspecto multilocular y ser confundido con un ameloblastoma. Además, también se presenta con mayor frecuencia en el maxilar inferior. De igual manera, el queratoquiste es más frecuente en el maxilar inferior, con relación a una pieza dentaria incluida, y puede también tener presentación uni o multilocular. El quiste odontogénico ortoqueratinizado se presenta en pacientes entre 20 y 40 años, en zona de molares, y el área radiolúcida es semejante también al quiste dentígero. La similitud radiográfica y clínica de estas lesiones obliga al radiólogo a realizar un adecuado diagnóstico diferencial, considerando la clínica y las características radiográficas, a fin de orientar un diagnóstico que permita su tratamiento $\left({ }^{40-42}\right)$.

\section{CONCLUSIÓN}

Si bien el diagnóstico definitivo del quiste dentígero es histopatológico, es fundamental el aporte del estudio imagenológico para su detección, diagnóstico y planificación de tratamiento. Actualmente, destaca la tomografía computarizada de haz cónico, la cual ha significado un notable avance para el radiólogo, al brindar información relevante para realizar un informe detallado y para el clínico al facilitar su decisión y planificación del tratamiento en beneficio del paciente.

También existen técnicas menos empleadas para diagnóstico del quiste dentígero, como la resonancia magnética, la cual presenta poca disponibilidad en la práctica diaria y es poco conocida como herramienta diagnóstica en estos casos. No obstante, los estudios realizados han entregado resultados promisorios. 
El odontólogo debe tener presente que estas nuevas tecnologías no reemplazan a las radiografías convencionales en 2D (a través de las cuales se realiza la detección y el diagnóstico inicial del quiste), representan si un importante complemento que debe considerarse cuando la condición clínica lo justifica, por ejemplo, en lesiones extensas que comprometen varias piezas dentarias y estructuras vecinas, o cuando se deba determinar con exactitud la relación de la lesión con la pieza incluida. También cuando sea relevante determinar la naturaleza y el contenido de la imagen observada, dado que el quiste dentígero es similar a otras patologías como el ameloblastoma uniquístico y queratoquiste, tanto a nivel clínico como radiográfico. En definitiva, las nuevas técnicas imagenológicas presentadas permiten complementar, precisar y facilitar el diagnóstico y tratamiento del quiste dentígero.

Contribución del autor: Rubén Castro Pesce ha participado en la revisión de datos e información para la redacción y aprobación de la versión final.

Fuente de financiamiento: Autofinanciada.

Conflicto de interés: El autor declara no tener conflicto de interés de ningún tipo. 


\section{REFERENCIAS BIBLIOGRÁFICAS}

1. Saad AY. Types, frecuency and distribution of 382 periapical lesions associated with endodontic therapy. J Md State Dent Assoc. 2007; 30 (1): 17-9.

2. Spini R, Bordino L, Cruz D, Martins A, Michalski J. Dentigerous cyst: a case report. Arch Argent Pediatr 2016; 114 (5): e 338-42.

3. Shear M, Speight PM. Cysts of the oral and maxilofacial regions. 4.a ed. Oxford: Munksgoard; 2007.

4. Ochsenius G, Escobar E, Godoy L, Peñafiel C. Odontogenic cysts: analysis of 2,944 cases in Chile. Med Oral Patol Oral Cir Bucal. 2007; 12 (2): 85-91.

5. Núñez-Urrutia S, Figueiredo R, Gay-Escoda C. Retrospective clinic-pathological study of 418 odontogenic cysts. Med Oral Patol Oral Cir Bucal. 2010; 15: 767-73.

6. Açikgöz, A, Uzun-Bulut E, Gündüz, K. Resumen de prevalencia y distribución de quistes odontogénicos y no odontogénicos en una población turca. Med Oral Patol Oral Cir Bucal 2012, 17 (3): 185-92.

7. Souza LB, Núñez MAG, Nonaka CFW, Medeiros MC, Torres TF, Emiliano GBG. Odontogenic cysts: demographic profile in a Brazilian population over a 38-year period. Med Oral Patol Oral Cir Bucal. 2010; 15: 583-90.

8. Donado M. Quistes de los maxilares y de la mandíbula En: Donado M. Cirugía bucal. Patología y técnica. 3.a Madrid: Masson; 2005.

9. Mosqueda T. New findings and controversies in odontogenic tumors. Med Oral Patol Oral Cir Bucal. 2008; 13: 555-8.

10. Urzúa R, Pinares J, Lobos N. Diagnóstico por imágenes de las lesiones benignas de los maxilares. Urba; 2017.

11. Kaplanl, Hirshberg A. The correlation between epithelial cell proliferation and inflammation in odontogenic kerato cyst. Oral Oncol. 2004; 40 (IO): 985-91.

12. Philipsen HP, Reichart PA, Ogawa I, Suei Y, Takata T. The inflammatory paradental cyst: A critical review of 342 cases from a literature survey, including 17 new cases from the author's files. J Oral Pathol Med. 2004; 33: 147-55.

13. Neville BW, Damm DD, Allen CM, Chi AC. Oral and maxillofacial pathology. St Louis: Elsevier; 2016.

14. Speignt PM, Takata T. New tumour entities in the 4 th edition of the World Health 8. Organization Classification of Head and Neck tumours: odontogenic and maxiIlofacial bone tumour. Virchows Arch. 2018; 472: 331-9.

15. Guerrero ME, Jacobs R, Loubele M, Schutyser F, Suetens P, Van Steenberghe D. Lo más avanzado en imágenes de TC de haz cónico para la planificación preoperatoria de la colocación del implante. Clin Oral Investig. 2006; 10 (1): 1-7. 
16. Hassan B, Van der Stelt P, Sanderink G. Exactitud de las mediciones tridimensionales obtenidas a partir de imágenes de tomografía computarizada de haz cónico para análisis cefalométrico: influencia de la posición de exploración del paciente. Eur J Orthod. 2009; 31 (2): 129-34.

17. Albarrán G, Gutiérrez R, Yépez Y. Marsupialización como tratamiento para quiste dentígero en un paciente pediátrico. Reporte de caso. Rev Odontol Andes. 2014; 9 (1): 47-55.

18. Shibata Y, Asaumi J, Yanagi Y, Kawai N, Hisatomi M, Matsuzaki H. Radiographic examination of dentigerous cysts in the transitional dentition. Dentomaxillofac Radiol. 2004; 33 (1): 17-20.

19. Narang R, Manchanda A, Arora P, Randhawa K. Dentigerous cyst of inflamatory origin- a diagnostic dilema. Ann Diagn Pathol. 2012; 16 (2): 119-23.

20. Konouchi H, Asaumi J, Yanagi Y, Hisatomi M, Arita E, Watanabe P. Diagnostic value of MR imaging for dentigerous cysts. Oral Radiol. 2014; 30 (1): 13-9.

21. Kouhsoltani M, Mesgarzadeh A, Moradzadeh M. Mandibular fracture associated with a dentigerous cyst: Report of a case and literatura review. J Dent Res Dent Clin Dent Prospects. 2015; 9 (3): 193-8.

22. Devi P, Thimmarasa V, Mehrotra V, Agarwal M. Multiple dentigerous cysts: A case report and review. J Maxillofac Oral Surg. 2015; 14 (1): 47-51.

23. Diaz JM, Puig LE, Ravinal II, Vives CB. Perfil epidemiológico, clínico y terapéutico de los quistes odontogénicos en Santiago de Cuba. Medisan 2014; 18 (1): 54.

24. El-Naggar A, Chan J, Grandis J, Takata T, Slootweg P. Who classification of head and neck tumours. International Agency for Research on Cancer: Lyon; 2016.

25. Mervyn, S, Speight, Paul. Cysts of the oral and maxillofacial regions. 4.a ed. Londres: Wiley; 2007.

26. Hasan S, Ahmed S, Reddy L. Dentigerous cyst in association with impacted inverted mesiodens: Report of a rare case with a brief review of literatura. Int $\mathrm{J}$ App Basic Med Res. 2014; 4 (1): 61-4.

27. Gallego Romero D, Torres Lagares D, García Calderón M, Romero Ruiz MM, Infante Cossío P, Gutiérrez Pérez JL. Diagnóstico diferencial y enfoque terapéutico de los quistes radiculares en la práctica odontológica cotidiana. Med Oral 2002; 7: 54-62.

28. Deana NF, Alves N. Cone Beam CT in Diagnosis and surgical planning of dentigerous cyst. Case Reports in Dentistry. 2017.

29. Kamburoglu K. Use of dentomaxillofacial cone beam computed tomography in dentistry. World J Radiol. 2015; 7 (6): 128-30.

30. Jing $G$, Jing $S$. Comparison between cone beam computed tomography and periapical radiography in the diagnosis of periapical disease. Hua Xi Kou Qiang Yi Xue Za Zhi. 2015; 33 (2): 209-13. 
31. Kamburoglu K. Use of dentomaxillofacial cone beam computed tomography in dentistry. World J Radiol. 2015; 7 (6): 128-30.

32. Figueiredo N, Alves N. Cone beam CT in diagnosis and surgical planning dentigerous cyst. Case Rep Dent. 2017.

33. Scarfe WC, Farman AG, Sukovic P. Aplicaciones clínicas de la tomografía computarizada de haz cónico en la práctica dental. J Can Dent Assoc. 2006; 72 (1): $75-80$.

34. Bonardi JP, Gómez PH, Momesso GA, Ferreira S, Pereira RD, Souza FA. Large dentigerous cyst associated to maxillary canine. J Craniofac Surg. 2017; 28 (1): e96-7.

35. Cassetta M, Di Carlo S, Pranno N, Stagnitti A, Pompa V, Pompa G. The use of high-resolution magnetic resonance on 3.0-T system in the diagnosis and surgical planning of intraosseous lesions of the jaws: preliminary results of a retrospective study. Eur Rev Med Pharmacol Sci. 2012; 16 (14): 2021-8.

36. Fujita M, Matsuzaki $H$, Yanagi $Y$, et al. Diagnostic value of MRI for odontogenic tumours. Dentomaxillofac Radiol. 2013; 42 (5).

37. Yifeng $\mathrm{H}$, Xindong $\mathrm{F}$, Zhenfeng $\mathrm{W}$. Diffusion-weighted MR imaging of unicystic odontogenic tumors for differentiation of unicystic ameloblastomas from keratocystic odontogenic tumors. Korean J Radiol. 2018; 19 (1): 79-84.

38. Tamashiro-Higa T, Mosqueda Taylor-A. Queratoquiste odontogénico en posición dentígera. Cir Ciruj 2005; 73: 127-31.

39. Martinelli-Kläy C, Martinelli C, Macedo H, Lombardi T. Unusual imaging features of dentigerous cyst: a case report. Dent J. 2019; 7 (3): e76.

40. Borrás J, Sánchez A, Gay C. Dentigerous cyst with parietal and intracystic calcifications: A case report and literatura review. J Clin Exp Dent. 2018; 10 (3): 296-9.

41. Chakraborty R, Sen S, Pandya D. "Two third tumor": A case report and its differential diagnosis. J Familiy Med Prim Care. 2019; 8 (6): 2140-3.

42. Shephard M, Coleman H. Simultaneous adenomatoid odontogenic and keratocystic odontogenic tumours in a patient with Gorlin-Goltz syndrome. Australian J Dent. 2014; 59: 121-4. 